(C) [2006] IEEE. Reprinted, with permission, from [Ying Yan, Jianguo Zhu, Youguang Guo and Haiwei Lu, Modeling and Simulation of Direct Torque Controlled PMSM Drive System Incorporating Structural and Saturation Saliencies, Industry Applications Conference, 2006. 41st IAS Annual Meeting. Conference Record of the 2006 IEEE (Volume:1 ) 8-12 Oct. 2006]. This material is posted here with permission of the IEEE. Such permission of the IEEE does not in any way imply IEEE endorsement of any of the University of Technology, Sydney's products or services. Internal or personal use of this material is permitted. However, permission to reprint/republish this material for advertising or promotional purposes or for creating new collective works for resale or redistribution must be obtained from the IEEE by writing to pubs-permissions@ieee.org. By choosing to view this document, you agree to all provisions of the copyright laws protecting it 


\title{
Modeling and Simulation of Direct Torque Controlled PMSM Drive System Incorporating Structural and Saturation Saliencie
}

\author{
Ying Yan, Jianguo Zhu, Youguang Guo and Haiwei Lu \\ Faculty of Engineering \\ University of Technology, Sydney \\ Sydney, Australia \\ yingyan@eng.uts.edu.au
}

\begin{abstract}
The direct torque controlled permanent magnet synchronous motor (PMSM) drive has become competitive compared with other types of drive systems because of its simple and sensorless control algorithm. The application of the system, however, is handicapped by the difficulty of starting under full load due to the unknown initial rotor position. This paper presents a nonlinear model of PMSMs which incorporates both the structural and saturation saliencies to enable the numerical simulation of rotor position detection algorithms. In this model, the phase inductances are expressed by Fourier series as the functions of the stator current and rotor position. The measurement of the inductances on a surface mounted PMSM is outlined. The simulation of direct torque control (DTC) on PMSM is conducted by using the proposed model, and the results are compared with that obtained by the PMSM model in the Simulink library. The performance of an initial rotor position estimation scheme with the model is simulated. Experiment is carried out based on the same algorithm to verify its validity. The effectiveness of the scheme to estimate the initial rotor position for an SPMSM is discussed and verified by numerical simulation.
\end{abstract}

Keywords- PMSM model; structural saliency; saturation saliency; inductance; direct torque control; initial rotor position estimation

\section{INTRODUCTION}

The direct torque controlled permanent magnet synchronous motor (PMSM) drive system has become competitive compared with other types of drive systems, because of its simple and sensorless control algorithm. The practical application of the system, however, is handicapped by the difficulty of starting under full load due to the unknown initial rotor position. A lot of effort has been made to detect the initial rotor position. Among them, the most versatile method is to make use of the structural and magnetic saturation saliencies which exist in the PMSM. The structural saliency can be employed to get the position of the rotor axis, while the saturation saliency, which is generated by the rotor permanent magnets, can be used to detect the magnetic polarity. Since the conventional PMSM model does not incorporate the saturation saliency, when developing a new method for the initial rotor position detection, it is not possible to numerically simulate the method, and the experimental trial and error method is widely.
This paper presents a nonlinear model of PMSMs which incorporates both the structural and saturation saliencies to enable the numerical simulation of new rotor position detection algorithms. In this model, the self and mutual differential inductances of the phase windings are expressed by Fourier series as functions of the stator current and rotor position. The measurement of the differential inductances is outlined in the paper. Based on the model, the direct torque control (DTC) scheme is simulated within Matlab/Simulink environment. To verify the validity of the new model, the simulation results from the model in the Simulink library are presented for comparison. Simulation of an initial rotor position estimation scheme based on the model is also conducted. Experiment is carried out to verify the correctness of the model and inductance measurement. Meanwhile, the possibility to detect the initial rotor position of a testing SPMSM with the scheme is investigated and analyzed by numerical simulation before being applied to a physical motor.

\section{NON-LINEAR MODEL OF PMSM WITH SALIENCIES}

\section{A. Flux Linkage and Circuit Equations}

In a PMSM, the field produced by the rotor magnets is the dominant component which determines the operating point of the magnetic core. Although the characteristics of the magnetic core is nonlinear, the magnetic circuit can be considered as piecewisely linearized around the operating point $P$ at a given rotor position. Thus, the total flux linkage of phase $a, \psi_{a}$, can be separated into two components [1-3]:

$$
\psi_{a}=\psi_{a s}\left(i_{m s}, \theta\right)+\psi_{a f}(\theta)
$$

where $\theta$ is the rotor position, $\psi_{a s}$ and $\psi_{a f}$ the flux linkage components produced by the magnetization component of the stator current $i_{m s}$, and rotor magnets, respectively. The flux linkage $\psi_{a s}$ can be further separated into components attributed by individual stator phase currents, $\psi_{a a}, \psi_{a b}$, and $\psi_{a c}$. Under the assumption of linearization, the flux linkage components can be considered as proportional to the corresponding currents: 


$$
\psi_{a}=\psi_{a a}+\psi_{a b}+\psi_{a c}+\psi_{a f}
$$

where $\psi_{a a}, \psi_{a b}$, and $\psi_{a c}$ are the flux linkage components of phase $a$ corresponding to each phase current. Under the assumption of linearization, the flux linkage components, $\psi_{a a}$, $\psi_{a b}$, and $\psi_{a c}$ can be considered as proportional to the corresponding phase currents:

$$
\begin{aligned}
& \psi_{a a}=L_{a a}\left(i_{a}, \theta\right) i_{a} \\
& \psi_{a b}=L_{a b}\left(i_{b}, \theta\right) i_{b} \\
& \psi_{a c}=L_{a c}\left(i_{c}, \theta\right) i_{c}
\end{aligned}
$$

The proportionality coefficients $L_{a a}, L_{a b}$, and $L_{a c}$, which are determined by the gradient of the magnetization curve at the operating point, are defined as the self and mutual incremental inductances of the phase $a$ winding. The flux linkages of phases $b$ and $c$ can be obtained similarly. The voltage equations of the three-phase stator windings can be written as:

$$
\left[\begin{array}{c}
v_{a} \\
v_{b} \\
v_{c}
\end{array}\right]=\left[\begin{array}{ccc}
R_{a} & 0 & 0 \\
0 & R_{b} & 0 \\
0 & 0 & R_{c}
\end{array}\right]\left[\begin{array}{c}
i_{a} \\
i_{b} \\
i_{c}
\end{array}\right]+\left[L_{T}\right] \frac{d}{d t}\left[\begin{array}{c}
i_{a} \\
i_{b} \\
i_{c}
\end{array}\right]+\left[\begin{array}{l}
e_{a f}+e_{a \theta} \\
e_{b f}+e_{b \theta} \\
e_{c f}+e_{c \theta}
\end{array}\right]
$$

where

$$
\left[L_{T}\right]=\left[\begin{array}{lll}
L_{a a}+i_{a} \frac{\partial L_{a a}}{\partial i_{a}} & L_{a b}+i_{b} \frac{\partial L_{a b}}{\partial i_{b}} & L_{a c}+i_{c} \frac{\partial L_{a c}}{\partial i_{c}} \\
L_{a b}+i_{a} \frac{\partial L_{a b}}{\partial i_{a}} & L_{b b}+i_{b} \frac{\partial L_{b b}}{\partial i_{b}} & L_{b c}+i_{c} \frac{\partial L_{b c}}{\partial i_{c}} \\
L_{a c}+i_{a} \frac{\partial L_{a c}}{\partial i_{a}} & L_{b c}+i_{b} \frac{\partial L_{b c}}{\partial i_{b}} & L_{c c}+i_{c} \frac{\partial L_{c c}}{\partial i_{c}}
\end{array}\right]
$$

$v_{a}, v_{b}$, and $v_{c}$ are the terminal voltages, $R_{a}, R_{b}$, and $R_{c}$ the winding resistances, and $i_{a}, i_{b}$, and $i_{c}$ the currents of phases $a, b$, and $c$, respectively, and $e_{a f}=\omega_{r} d \psi_{a f} / d \theta, \quad e_{b f}=\omega_{r} d \psi_{b f} d d \theta$, and $e_{c f}=\omega_{r} d \psi_{c f} d \theta$ are the electromotive forces (emfs) induced by the rotor magnets, $\omega_{r}=d \theta / d t$ is the mechanical angular speed of the rotor, and

$$
\begin{aligned}
& e_{a \theta}=\omega_{r}\left(i_{a} \frac{\partial L_{a a}}{\partial \theta}+i_{b} \frac{\partial L_{a b}}{\partial \theta}+i_{c} \frac{\partial L_{a c}}{\partial \theta}\right)(8) \\
& e_{b \theta}=\omega_{r}\left(i_{a} \frac{\partial L_{a b}}{\partial \theta}+i_{b} \frac{\partial L_{b b}}{\partial \theta}+i_{c} \frac{\partial L_{b c}}{\partial \theta}\right)(9)
\end{aligned}
$$

$$
e_{c \theta}=\omega_{r}\left(i_{a} \frac{\partial L_{a c}}{\partial \theta}+i_{b} \frac{\partial L_{b c}}{\partial \theta}+i_{c} \frac{\partial L_{c c}}{\partial \theta}\right)(10)
$$

the emfs induced by the variation of flux linkage due to the saliencies.

\section{B. Electromagnetic Torque}

The electromagnetic torque of the PMSM can be obtained by taking the partial derivative of the system co-energy with respect to the rotor angle, i.e. $T=\partial W_{f}^{\prime} / \partial \theta$, and it can be derived as:

$$
\begin{aligned}
T= & \frac{\partial \psi_{a f}}{\partial \theta} i_{a}+\frac{\partial \psi_{b f}}{\partial \theta} i_{b}+\frac{\partial \psi_{c f}}{\partial \theta} i_{c}+\frac{\partial L_{a a}}{\partial \theta} \frac{i_{a}^{2}}{2}+\frac{\partial L_{b b}}{\partial \theta} \frac{i_{b}^{2}}{2}+\frac{\partial L_{c c}}{\partial \theta} \frac{i_{c}^{2}}{2} \\
& +\frac{2 \partial L_{a b}}{\partial \theta} i_{a} i_{b}+\frac{2 \partial L_{a c}}{\partial \theta} i_{a} i_{c}+\frac{2 \partial L_{b c}}{\partial \theta} i_{b} i_{c}(11)
\end{aligned}
$$

The electromagnetic torque has two components: one produced by the stator currents and rotor magnets, and the other by the saliencies.

\section{NONLINEAR INCREMENTAL INDUCTANCE}

\section{A. Analytical Expression of Nonlinear Inductance}

As discussed above, the structural and magnetic saturation saliencies can be reflected by the variation of the self and mutual inductances of the stator windings. These inductances are nonlinear functions of stator phase winding currents and the rotor position, which require a large amount of data to describe numerically. Use of analytical expressions of these nonlinear inductances could simplify the implementation of the numerical simulation model and reduce significantly the amount of parameters.

Since the phase inductance is a periodic function of rotor angular position, the relationship between the phase inductance and the rotor position can be expressed by a Fourier series [4]:

$$
L(\theta)=a_{0}+\sum_{m=1}^{n}\left(a_{m} \cos m \theta+b_{m} \sin m \theta\right)
$$

The number of terms of the Fourier series can be chosen through the best curve fitting of the experimentally measured phase inductances.

Due to the nonlinear characteristics of the magnetic core, the inductances also vary with the stator current. Consequently, different sets of coefficients, $a_{0}, a_{m}$, and $b_{m}(m=1,2, \ldots, n)$, can be obtained with different currents, and expressed as functions of corresponding currents. Therefore, we have:

$$
L(i, \theta)=a_{0}(i)+\sum_{m=1}^{n}\left(a_{m}(i) \cos m \theta+b_{m}(i) \sin m \theta\right)
$$


For a group of known values of phase inductance, $L\left(\theta_{j}, i_{k}\right)$, the coefficients of the corresponding Fourier series can be obtained by nonlinear curve fitting with sufficient accuracy, where $j$ and $k$ refer to the various experimental rotor positions and currents, respectively. The nonlinear model of the self- and mutual-inductances can be readily incorporated into the PMSM model presented in Section II.

\section{B. Experimental Measurement of Phase Inductances}

When a PMSM is in its normal operating state, the total flux is composed of both the stator and rotor fluxes, $\left(\psi_{t}=\right.$ $\psi_{r}+\psi_{s}$ ). The influence of the stator flux $\psi_{s}$ cannot be neglected. Therefore, the phase inductance expression will be affected by the rotor position and stator flux.

In order to estimate the saturation effect at various stator fluxes, DC offsets, $i_{d c}$, are used to emulate the effect of the three-phase currents. Based on the analysis of the relationship between the inductance variation and the saturation of magnetic core, a method for measuring the phase inductances is designed and the saturation effect can be reflected in the inductance expression measured by this method.

The experiment is carried out on a 6-pole SPMSM, and the rotor of the motor is locked by a dividing head as shown in Fig.1. Various DC offsets are injected to one of the stator windings. Meanwhile, a small AC current is applied to one phase while the other two are open-circuited. The power, voltage, and current of the excited phase are measured and the voltages of the other two phases are also recorded. The self and mutual inductances of phases $a, b$ and $c$ at different rotor positions and stator fluxes can then be calculated via circuit analysis. Fig. 2 illustrates the self-inductances of phase $a, L_{s s a}$, at various rotor positions with $6 A \mathrm{DC}$ offsets and without DC offsets.

According to the magnetic flux distribution in a PMSM, it can be concluded that the inductances by considering the structural and saturation saliencies are functions of amplitudes and angles of both stator and rotor fluxes, $L\left(\theta_{s}, \theta_{r},\left|\psi_{s}\right|,\left|\psi_{r}\right|\right)$, where $\theta_{s}$ is the position of the stator flux, $\theta_{r}$ the position of rotor flux, and $\left|\psi_{s}\right|$ and $\left|\psi_{r}\right|$ are the amplitudes of the stator and rotor fluxes. Since the amplitude of the rotor flux due to the permanent magnets can be considered as a constant, the inductances can be simplified as functions of $\theta_{s}, \theta_{r}$, and $\left|\psi_{s}\right|$, and rewritten as $L\left(\theta_{s}, \theta_{r},\left|\psi_{s}\right|\right)$. Moreover, due to the symmetrical distribution of three phase stator windings, the three phase inductances have the same profile with a $\pm 120^{\circ}$ phase shift.

In order to simplify the inductance expressions, the angle between the stator flux, $\psi_{s}$, and the stator winding axis of phase $a$, denoted by $\alpha$, and the angle between the stator and rotor fluxes, denoted by $\delta$, as shown in Fig. 3, are used in the inductance model instead of $\theta_{s}$ and $\theta_{r}$. Because of the symmetrical motor structure, the inductances at the following positions can be obtained by injecting various DC currents to phase $a$ according to the previous experiment: (1) $\alpha=0^{\circ}, \delta=0^{\circ}$ $360^{\circ}$, and $\left|\psi_{s}\right|$ corresponding to $i_{m s}=0 A-I_{\text {rated }}$ (the rated current); (2) $\alpha=120^{\circ}, \delta=0^{\circ}-360^{\circ}$, and $\left|\psi_{s}\right|$ corresponding to $i_{m s}=0 A-I_{\text {rated }}$; (3) $\alpha=240^{\circ}, \delta=0^{\circ}-360^{\circ}$, and $\left|\psi_{s}\right|$ corresponding to $i_{m s}=0 A-I_{\text {rated }}$. The phase $a$ inductance, $L_{s a a}\left(\alpha, \delta, i_{m s}\right)$, at any stator and rotor fluxes $\left(\alpha=0^{\circ}-360^{\circ}, \delta=0^{\circ}-360^{\circ}\right.$, and $\left.i_{m s}=0 A-I_{\text {rated }}\right)$ can be estimated by linear interpolation of the measured inductance expression. Due to the symmetry of the three phase windings, the inductances of phases $b$ and $c$ at any rotor position and stator current can be estimated based on the phase $a$ inductance expression by shifting the rotor position by $\pm 120^{\circ}$, respectively. At a given $\delta$ and $i_{m s}$, we have $L_{s b b}\left(\alpha, \delta, i_{m s}\right)=$ $L_{\text {saa }}\left(\alpha-120^{\circ}, \delta, i_{m s}\right)$, and $L_{s c c}\left(\alpha, \delta, i_{m s}\right)=L_{s a a}\left(\alpha+120^{\circ}, \delta, i_{m s}\right)$. Similar procedure can be applied to estimate the mutual inductances. Therefore, the structural and saturation saliencies of the motor magnetic filed could be mapped out.

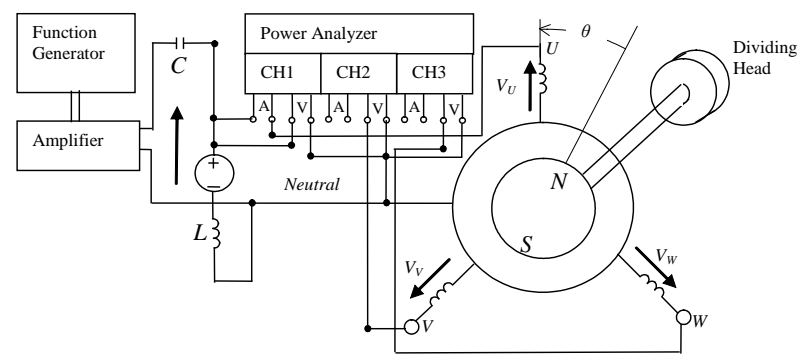

Figure 1. Experimental setup for inductance measurement

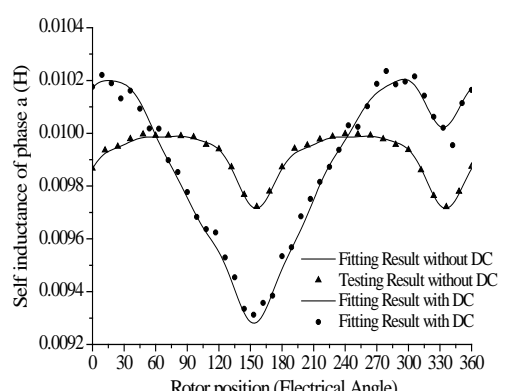

Figure 2. Measured self inductances with and without DC offset and fitting curves

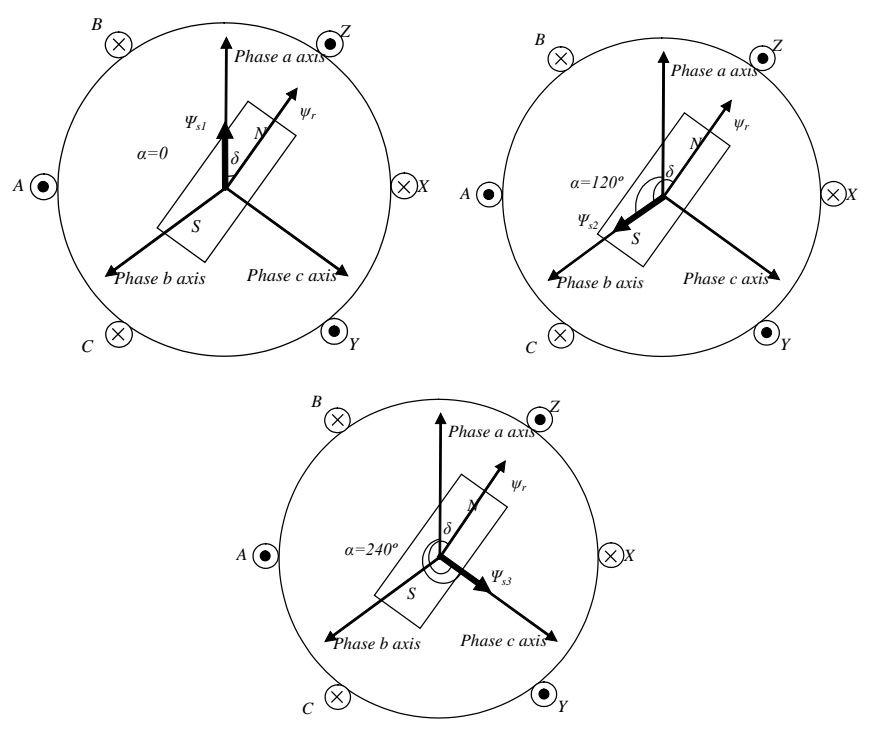

Figure 3. (a) Rotor and stator fluxes linking phase $a$ with a DC offset in phase $a$; (b) Rotor and stator fluxes linking phase $b$ with a DC offset in phase $a$; (c) Rotor and stator fluxes linking phase $c$ with a DC offset in phase $a$. 


\section{NUMERICAL SimUlation OF DTC DRIVE}

\section{A. Implementation of PMSM DTC System}

Fig.4 shows a schematic block diagram of the conventional DTC [5]. In the DTC of PMSM, the electromagnetic torque can be controlled by keeping the amplitude of the stator flux linkage constant and controlling the load angle by applying a proper stator voltage vector. The actual flux and torque values are firstly calculated based on the proposed PMSM model by considering saliencies, and in the hysteresis comparators, the calculated torque/flux are compared with the torque/flux references. The outputs of the comparators are used to select the proper stator voltage vector based on the optimal voltage vector switching logic so as to minimize the errors between the actual and reference values of flux and torque. In the conventional DTC, only six voltage vectors and two zerovoltage vectors can be produced by a two level voltage-source inverter.

The PMSM DTC scheme shown in Fig.4, which is a closed-loop system with an outer speed control loop and an inner torque control loop, is implemented using Simulink package. In the simulation model, the two-phase stationary $\alpha-\beta$ reference frame is used. The stator currents $i_{a}, i_{b}$ and $i_{c}$ and the DC-link voltage $V_{d c}$ are detected by current and voltage transducers. The currents are then transformed by the Clark transformation into components $\left(i_{\alpha}, i_{\beta}\right)$, and the $u_{\alpha}$ and $u_{\beta}$ are obtained by the combination of $u_{d c}$ and the switching state in the previous sampling period. The stator flux linkage and the electromagnetic torque are obtained as follows:

$$
\begin{gathered}
\lambda_{\alpha}\left(t_{n+1}\right)=\left(u_{\alpha}\left(t_{n}\right)-R_{s} i_{\alpha}\left(t_{n}\right)\right) T_{s}+\lambda_{\alpha}\left(t_{n}\right) \\
\lambda_{\beta}\left(t_{n+1}\right)=\left(u_{\beta}\left(t_{n}\right)-R_{s} i_{\beta}\left(t_{n}\right)\right) T_{s}+\lambda_{\beta}\left(t_{n}\right) \\
\lambda_{s}\left(t_{n}\right)=\sqrt{\lambda_{\alpha}^{2}\left(t_{n}\right)+\lambda_{\beta}^{2}\left(t_{n}\right)} \\
T_{e m}\left(t_{n}\right)=\frac{3}{2} P\left(\lambda_{\alpha} i_{\beta}-\lambda_{\beta} i_{\alpha}\right)
\end{gathered}
$$

\section{B. Simulation by the Proposed Model}

Based on the proposed model and the parameters identified by experiments, the dynamic performance of a PMSM DTC drive system with known initial rotor position is simulated. Figs. 5 and 6 show the simulated stator current, and the stator flux trajectory of the system corresponding to a speed command of $1500 \mathrm{rpm}$ and $4.5 \mathrm{Nm}$ load torque, with the parameters of the speed loop PI controller carefully tuned for optimal performance.

\section{Comparison with PMSM Model in Similink Library}

The simulation results of the proposed PMSM model are compared with that of the PMSM model in the Simulink library with constant inductances under the same conditions. Figs.7 and 8 show the simulated rotor speed and electromagnetic torque of these two models in the same time frame.

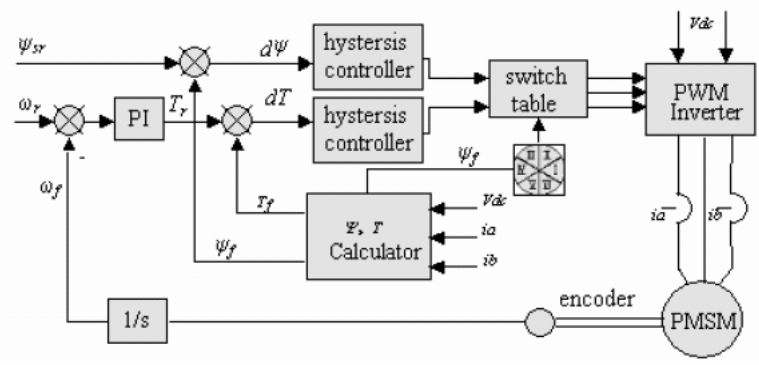

Figure 4. Control block diagram of the conventional DTC

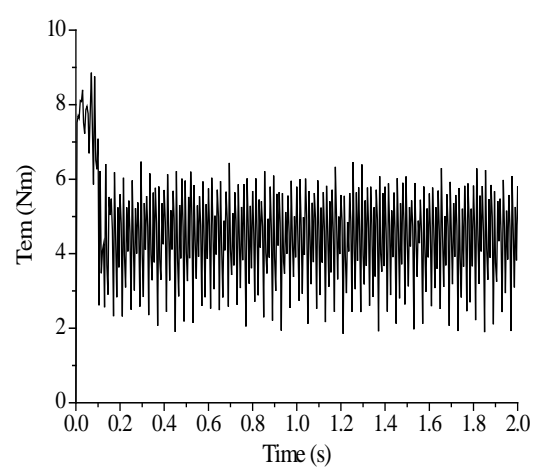

Figure 5. Electromagnetic torque

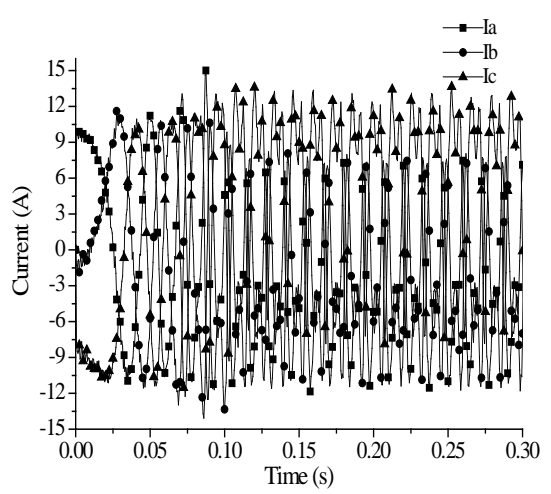

Figure 6. Stator currents by the proposed model

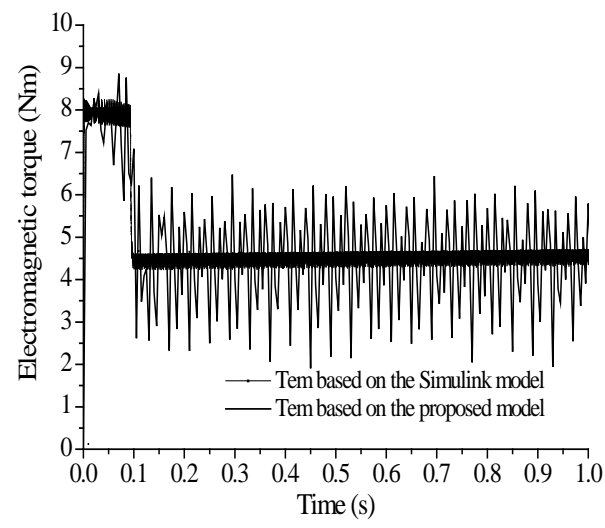

Figure 7. Torque comparison between the two models 


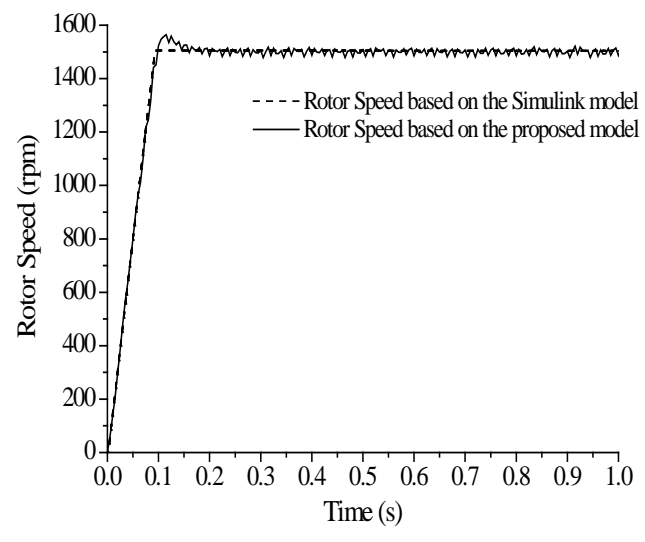

Figure 8. Speed comparison between the two models

\section{Estimation OF INITIAL ROTOR POSITION}

\section{A. Experimental Procedure}

In the DTC scheme, the real time rotor position is derived by integrating the back emf against time. However, the starting procedure under full load is very difficult because no current or voltage information is available for determining the rotor position before starting. Therefore, it is essential to develop a sensorless method to determine the initial rotor position for full load starting of PMSM drive systems. Numerous approaches have been proposed to estimate the initial rotor position from motor's terminal properties. Most of them are effective for the motor with large structural saliency, e.g. the interior PMSM. However, in a SPMSM, it becomes difficult due to the small saliencies. The saturation saliency may have to be used because the structural saliency is not significant.

It has been discussed that the winding inductance is a function of rotor position and stator current. With this feature, the initial rotor position can be estimated by injecting two kinds of voltages pulse with high and low amplitude to the stator winding terminals, respectively. Both of the voltages pulses are applied as the following [6]. At an unknown initial rotor position, a positive and two negative pulses are applied to each phase. After the voltage pulse for one phase, a voltage pulse in the opposite direction is provided to turn the phase current to zero, as shown in Fig.9 [7].

In the experiment, the pulse voltages are produced by the PWM inverters, and the gate signals of the inverters are controlled by dSPACE DS1104 system. For example, when the positive pulse is applied to phase $A$, the gate signals of 100 are created and applied to the inverters. In this case, the equivalent circuit of the motor at standstill and the gate pattern of 100 are shown in Fig.10.

\section{B. Experimental Results}

The high and low voltage pulses are applied to a 6-pole SPMSM from an inverter. The corresponding phase currents are measured by current probe and oscilloscope. Figs. 11-12 show the experimental current waveform. In Fig.11, the three phase current response is recorded for high voltage pulse with the state of 100, 010 and 001 respectively. In Fig.12, the current response is obtained for low voltage pulse with the state of 100. For each phase, the measurement is taken at every locked rotor position and the motor is mechanically rotated from one position to the next. The measured three phase peak currents at different rotor positions with high and low voltage pulses are plotted in Fig. 13 (a) and (b).

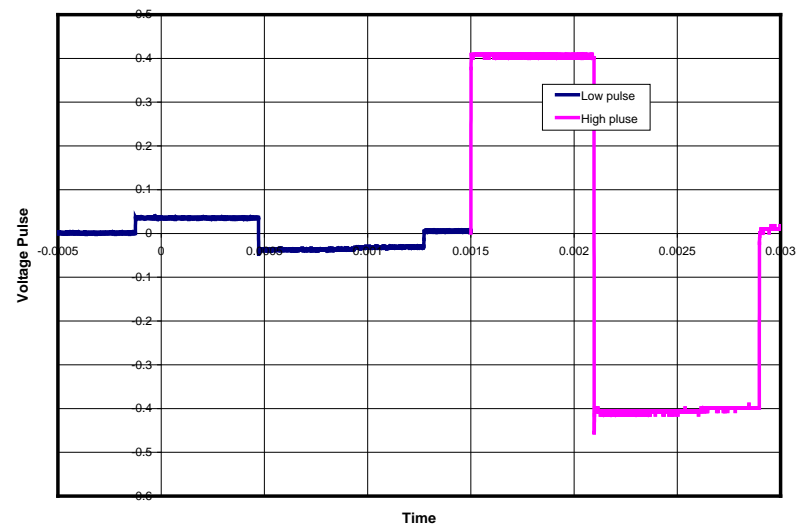

Figure 9. Voltage pulse

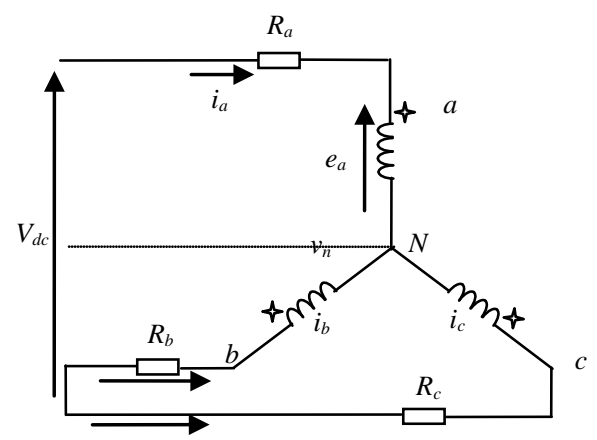

Figure 10. Equivalent circuit of the motor at standstill

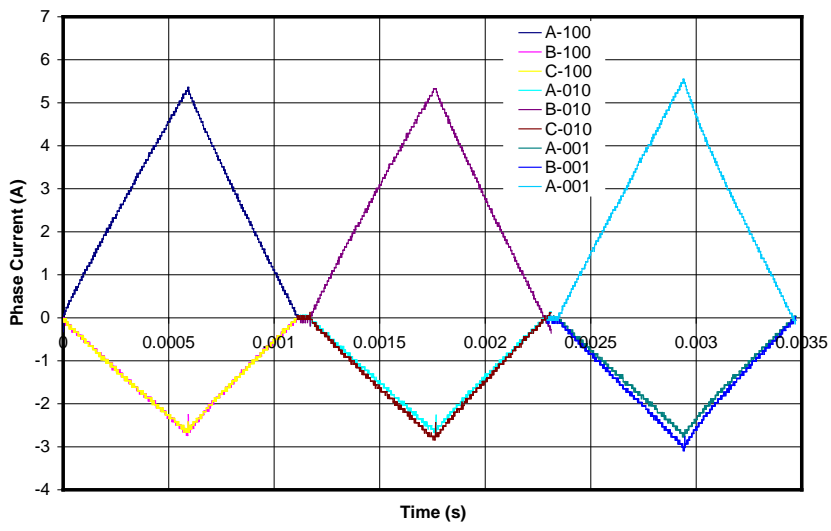

Figure 11. Experimental current response to high voltage pulse 


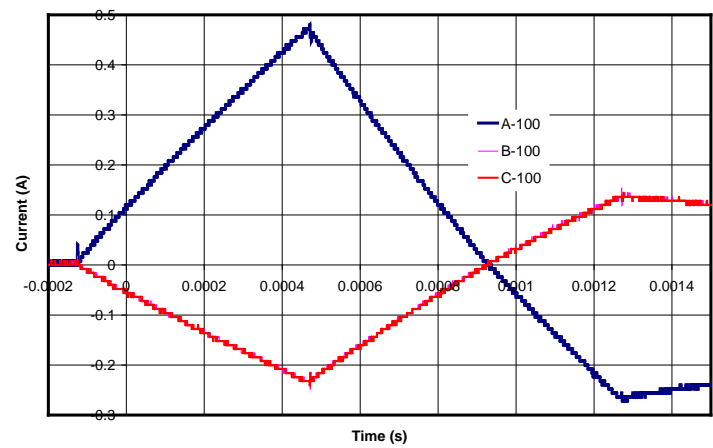

Figure 12. Experimental current response to low voltage pulse

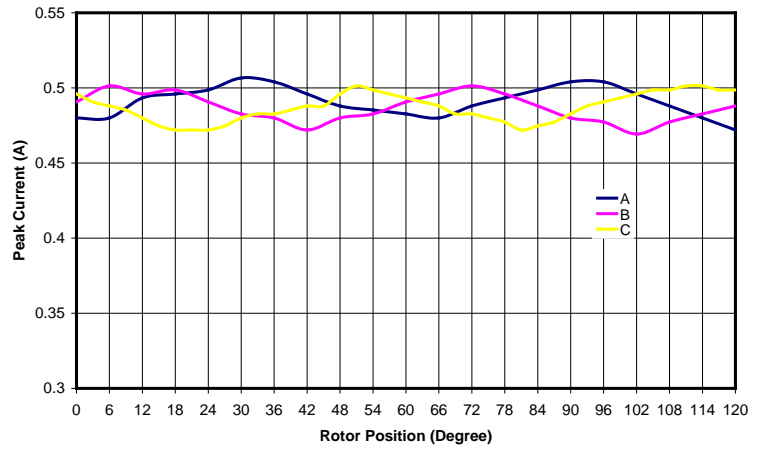

(a)

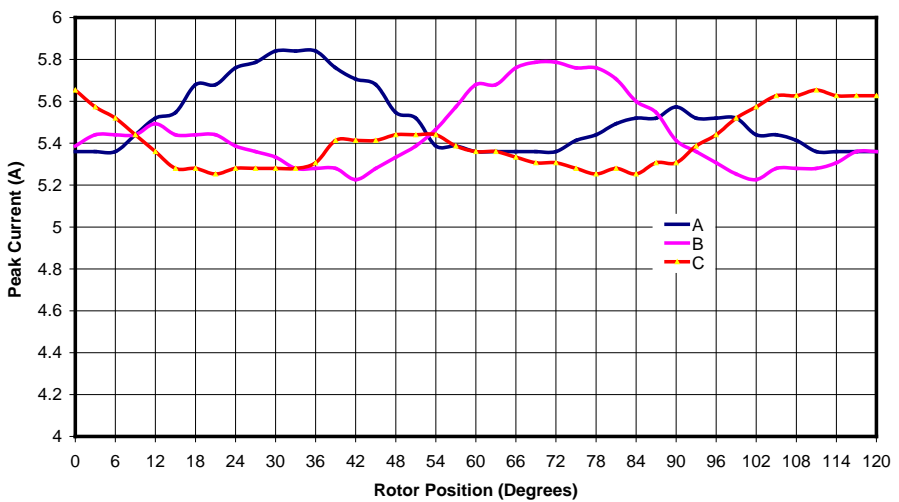

(b)

Figure 13. Peak current versus rotor position: (a) with low voltage pulse, (b) with high voltage pulse

\section{Comparison of peak current and inductance variation}

Compared with the almost sinusoidal waveform at the nonsaturated condition, it can be seen the magnetic saturation due to the stator currents could be neglected with the low voltage pulse. However, with the high voltage pulse, the magnetic saturation becomes evident.

It has known that the phase inductances are functions of rotor positions and stator currents, because the field produced by the stator currents change the field distribution produced by the rotor magnet. At some positions, magnetic core is more saturated by the stator flux and the inductance of the stator windings is reduced. Therefore the smallest inductance might happen. While at other positions, magnetic core is less saturated and the inductance might become larger. Since the inductance variation has been measured, it is possible to analyse the relation between the inductance variation and peak current variation at various rotor positions.

The phase self inductance variation with DC offsets of $6 \mathrm{~A}$ at various rotor positions, which reflects the saturation saliency due to DC offsets, are compared with currents with high voltage pules as shown in Figs.14-16. The inductance variation without DC offset is also plotted to demonstrate the structural saliency of the motor and aid to identify the $d$-and $q$-axis of the rotor. It can be seen that the structural saliency is very small due to the surface mounted structure of the testing motor.

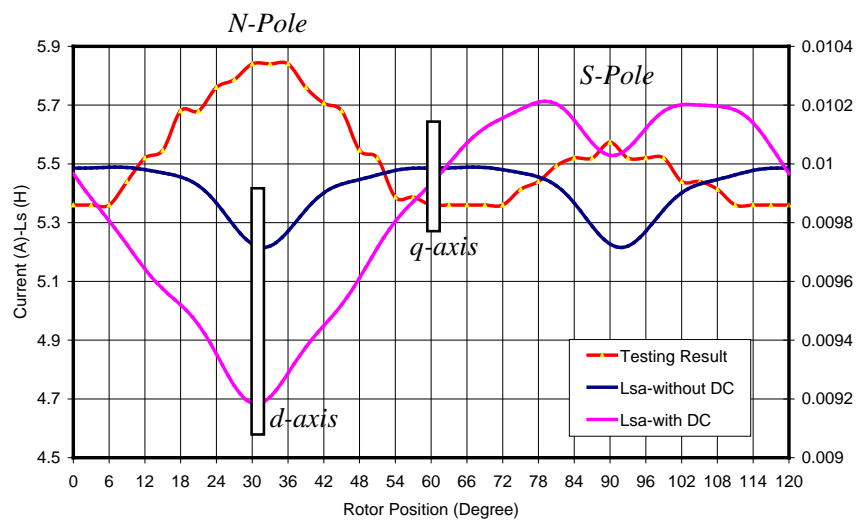

Figure 14. Phase $A$ inductance and peak current variation

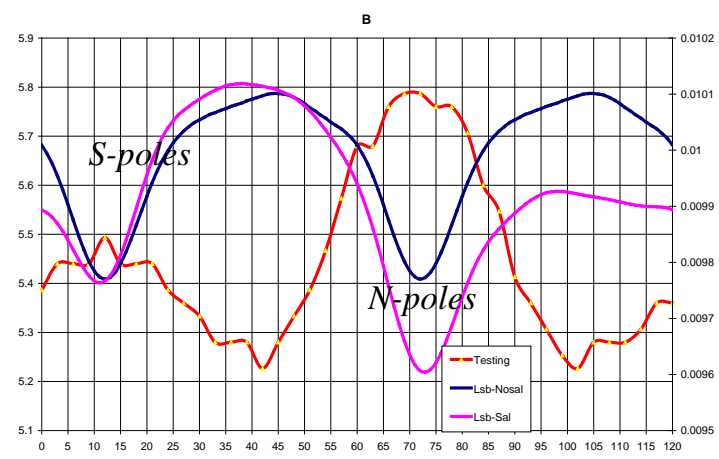

Figure 15. Phase $B$ inductance and peak current variation

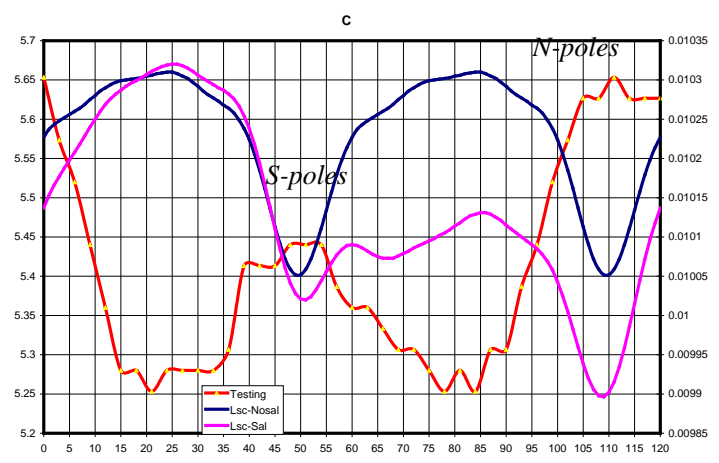

Figure 16. Phase $C$ inductance and peak current variation 
In Figs. 14-16, the inductance variations of phase $A, B$ and $C$ in one electrical cycle of the rotor position are plotted out. From the figures, a clear difference between the inductance in the $d$ - and $q$-direction has been detected. For the SPMSM, the $q$-axis inductance is higher than $d$-axis inductance, which can be used to determine the $d$ - and $q$-axis. When DC offset is applied to phase windings, the inductance becomes lower or higher when the rotor aligns with the $d$-axis of the rotor, compared with the results without DC offset. Therefore, the positions of $d$ - and $q$-axis are identified, and indicated in the figures. Meanwhile, the difference between north and south poles of the rotor could also be obtained from the inductance with DC offsets. The flux due to stator currents in the north pole direction will drive the magnetic core into more saturated and the inductance will become even lower. While the magnetic field will become less saturated with an opposite direction flux due to stator current under the south pole [8]. It provides a possibility to detect the north and south poles.

For peak current variations, the positions of $d$ - and $q$-axis could also be detected by analysing the saturation saliency caused by high voltage pules. At the position which aligns with the rotor $d$-axis under the north pole, the maximum current is detected, which corresponds to minimum inductance because of $d i / d t=V / L$. The minimum current value occurs at the position under the south pole, as shown in Figs. 14-16.

It can be concluded that both the structural and saturation saliencies can be reflected from inductance variations and verified by the peak current profile. Based on the previous model and measured inductance, simulations of the initial rotor position estimation scheme are carried out.

\section{Comparison of simulated and measured peak currents}

With the equivalent circuit in Fig.10, dynamic simulation of voltage pulse injection algorithm is carried out. A Simulink model of the motor at standstill is built. The measured inductances at various rotor positions are used as parameters of the model. High voltage pulse is injected to different phases. The peak currents of each phase corresponding to various rotor positions are plotted in Fig.17.

Generally, the variation trend of the simulation and experimental peak currents is similar. As it has been discussed, the self and mutual inductances were measured only at limited rotor positions and stator currents. The inductances at other positions are calculated by linear interpolation. That means the inductance values at those positions are roughly estimated and not accurate enough. Error of the simulation results based on the inductances might be large at some specific positions.

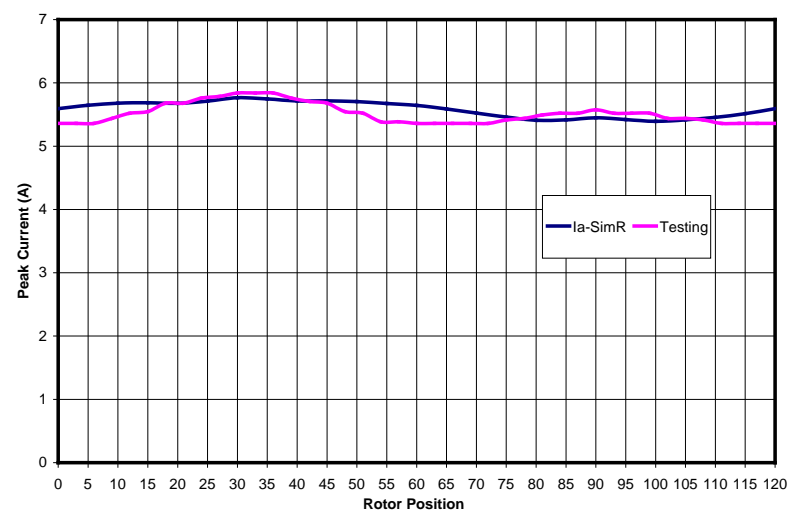

Figure 17. Comparsion of the simulated and measured peak currents

\section{E. Initial rotor position estimation algorithm}

Based on the above analysis, it is possible to identify the $d$ and $q$-axis and polarity of the rotor from the measured peak current profile. The peak current variation with low voltage pulses in Fig.13 (a) is very small due to the surface mounted structure. The peak current distribution is ideally regarded as sinusoidal function of the rotor position and can be modeled as:

$$
\begin{aligned}
& I_{a}=I_{0}+\Delta I_{0} \cos (2 \theta) \\
& I_{b}=I_{0}+\Delta I_{0} \cos \left(2 \theta+\frac{2 \pi}{3}\right) \\
& I_{c}=I_{0}+\Delta I_{0} \cos \left(2 \theta-\frac{2 \pi}{3}\right)
\end{aligned}
$$

where $I_{a}, I_{b}$ and $I_{c}$ are peak currents and $\theta$ rotor position. $I_{0}$ is obtained by $I_{0}=\left(I_{a}+I_{b}+I_{c}\right) / 3$ and $\Delta I_{0}$ could be obtained by curve fitting of the experimental results. The curve fitting of the experimental results are shown in Fig. 18 (a). As it has been discussed in [6], the signs of $\Delta I_{a}, \Delta I_{b}$, and $\Delta I_{c}$ could be used to determine region of the rotor position. Although the region of rotor position could be estimated by the simulation results of $\Delta I_{a}, \Delta I_{b}$, and $\Delta I_{c}$, it is almost impossible to implement it in real time system for SPMSM because the obtained saliency is quite small.

As for the peak current variation measured with high voltage pulses, the phase currents as shown in Fig.13 (b) could be also modeled as an average value, $I_{0}$, plus some offset value $\Delta I_{01}$ and $\Delta I_{02}$, as a function of rotor positions, $\theta$, as followings:

$$
\begin{gathered}
I_{a}=I_{0}+\Delta I_{01} \cos (\theta)+\Delta I_{02} \cos (2 \theta) \\
I_{b}=I_{0}+\Delta I_{01} \cos \left(\theta-\frac{2 \pi}{3}\right)+\Delta I_{02} \cos \left(2 \theta+\frac{2 \pi}{3}\right) \\
I_{c}=I_{0}+\Delta I_{01} \cos \left(\theta+\frac{2 \pi}{3}\right)+\Delta I_{02} \cos \left(2 \theta-\frac{2 \pi}{3}\right)
\end{gathered}
$$

Curve fitting is carried out based on the experimental results. The fitting results are plotted in Fig. 18 (b). The 
following algorithm for initial rotor position detection is presented. The peak current profiles of phases $A, B$ and $C$ at various rotor positions have been stored as a look up table. High voltage pulse as described before are injected to various phases. The phase current responses, $I_{a}, I_{b}$ and $I_{c}$ are measured and peak currents are obtained. For phase $A$, the rotor positions corresponding to the peak current $I_{a p}$ could be identified, such as $\theta_{a 1} \theta_{a 2}, \theta_{a 3}$ and so on. For phase $B$ and $C$, the rotor positions corresponding to $I_{b p}$ and $I_{c p}$ could be obtained, such as $\theta_{b 1} \theta_{b 2}$, $\theta_{b 3}, \theta_{c 1} \theta_{c 2}, \theta_{c 3}$ and so on. There must be only one angle with the same value for all the angles. For example, if we have $\theta_{a 1}=$ $\theta_{b 3}=\theta_{c 2}, \theta_{a 1}$ is the estimated rotor angle. Fig. 19 shows the simulation results of the algorithm. By this voltage pulse injection technique, both the position of $d$-axis and the north or south pole of the rotor could be estimated.

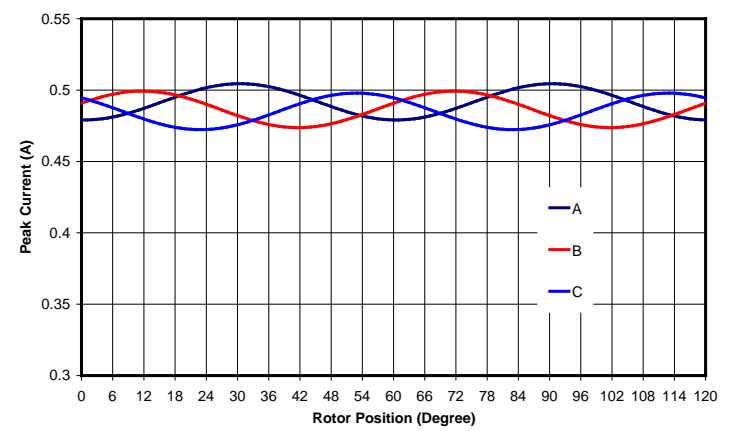

(a)

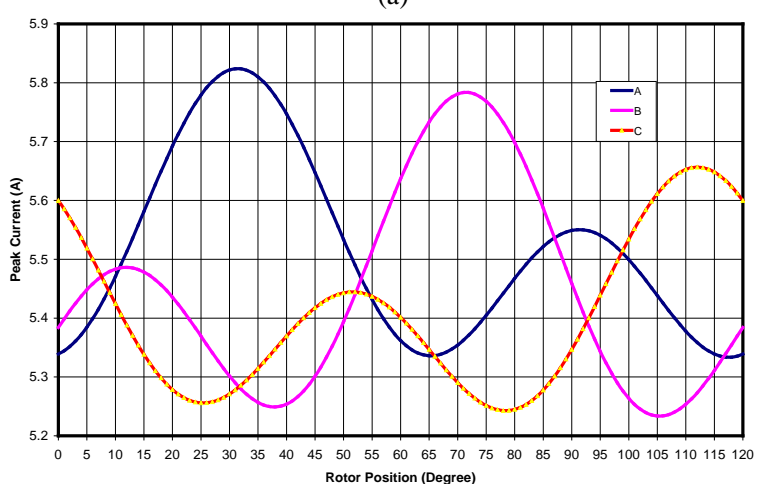

(b)

Figure 18. Fitting peak current to high and low voltage pulses

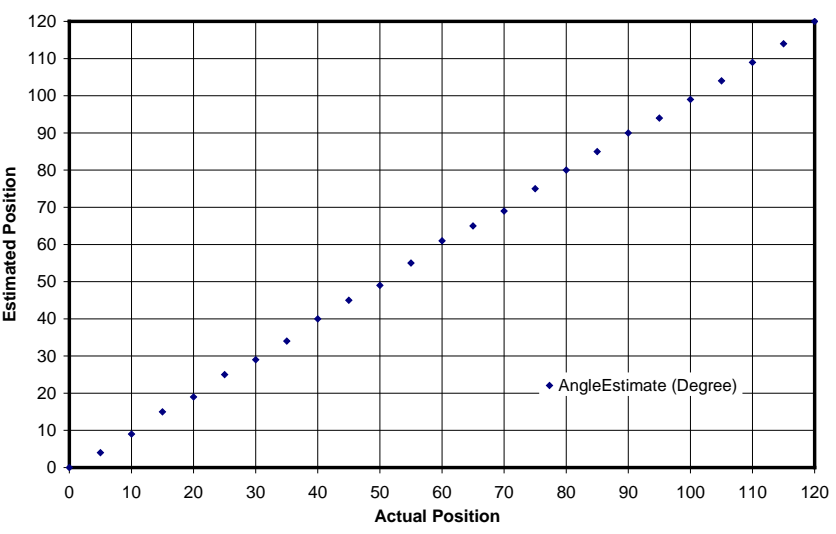

Figure 19. Simulation result of position estimation

\section{CONCLUSIONS}

A nonlinear PMSM model incorporating both the structural and magnetic saturation saliencies has been presented in this paper. In the model, the saliencies are reflected by the variation of the stator winding inductances with respect to the rotor positions and stator currents. The nonlinear inductances at various rotor positions and stator currents are discussed and experimentally obtained from a surface mounted PMSM. The Fourier series is employed to describe the relationship between the inductance and the rotor position and stator currents.

Dynamic simulation of a PMSM drive system with the conventional DTC scheme using the derived model is conducted and the results are compared with that obtained by the PMSM model in the Simulink library.

The performance of an initial rotor position estimation scheme based on the model is simulated. Experiment with the same algorithm is carried out to verify its validity. The effectiveness of the scheme to estimate the initial rotor position for an SPMSM could be assessed and analyzed within Simulink environment before it is implemented in real time system.

\section{REFERENCES}

[1] Ying Yan, Jianguo Zhu, and Haiwei Lu, “A PMSM Model Incorporating Structural and Saturation Saliencies”, the $8^{\text {th }}$ International Conference on Electrical Machines and Systems (ICEMS), Nanjing, China, 27th - 29th September 2005.

[2] Ying Yan, Jianguo Zhu, and Haiwei Lu, "Direct Torque Control of a Surface-Mounted Permanent Magnet Synchronous Motor based on Accurate Modelling”, the Australasian Universities and Power Engineering Conference (AUPEC), Hobart, Australia., 25th - 28th September 2005.

[3] Y. Yan, J.G. Zhu, H.W. Lu, Y.G. Guo, and S.H. Wang, "Study of a PMSM model incorporating structural and saturation saliencies," in Proc. $6^{\text {th }}$ IEEE International Conference on Power Electronics and Drive Systems, Kuala Lumpur, Malaysia, Nov. 28 - Dec. 1, 2005, pp. 575-580.

[4] P. Cui, J.G. Zhu, Q.P. Ha, G.P. Hunter, and V.S. Ramsden, "Simulation of non-linear switched reluctance motor drive with PSIM," in Proc. 5th Int. Conf. on Electrical Machines and Systems, Aug. 2001, vol. 1, pp. 1061-1064.

[5] D. Sun, "High performance direct torque control for permanent magnet synchronous motors,” Ph.D. Thesis, Zhejiang University, P.R. China, March, 2004

[6] N. Matsui and T. Takeshita, "A novel starting method of sensorless salient-pole brushless motor," Conference Record of the 1994 IEEE Industry Applications Society Annual Meeting, Oct. 2-6, 1994, vol.1, pp. 386-392.

[7] P.B. Schmidt, M.L. Gasperi, G. Ray, and A.H. Wijenayake, "Initial rotor angle detection of a nonsalient pole permanent magnet synchronous machine," Conference Record of the 1997 IEEE Industry Applications Society Annual Meeting, Oct. 5-9, 1997, Vol.1, pp. 459-463.

[8] S. Ostlund, and M. Brokemper, "Initial rotor position detections for an integrated PM synchronous motor drive", Conference Record of the 1995 IEEE Industry Applications Society Annual Meeting, 8-12 Oct. 1995, Vol.1, pp. 741 - 747. 